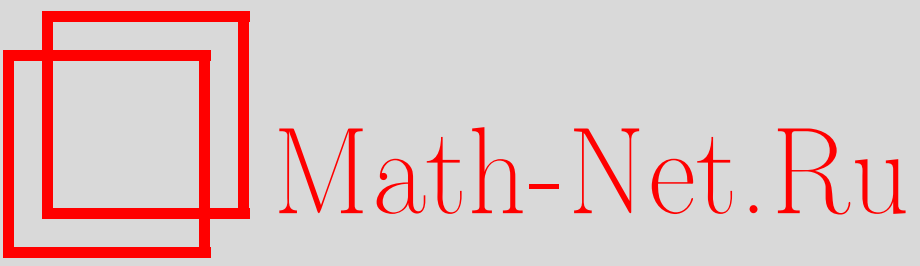

В. В. Жаринов, Когомологии Хохшильда алгебры гладких функций, ТМФ, 2004, том 140, номер 3, 355-366

DOI: https://doi.org/10.4213/tmf107

Использование Общероссийского математического портала Math-Net.Ru подразумевает, что вы прочитали и согласны с пользовательским соглашением

http://www.mathnet.ru/rus/agreement

Параметры загрузки:

IP : 54.157 .27 .8

26 апреля 2023 г., 13:58:06

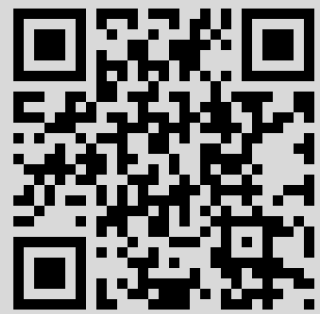


ТЕОРЕТИЧЕСКАЯ

И МАТЕМАТИЧЕСКАЯ

ФИЗИКА

Том 140, № 3

сентябрь, 2004

(C) 2004 г.

В.В. Жаринов*

\section{КОГОМОЛОГИИ ХОХШИЛЬДА АЛГЕБРЫ ГЛАДКИХ ФУНКЦИЙ}

Опираясь на развитую ранее технику, использующую преобразование Лапласа обобщенных функций с компактным носителем, вычислены когомологии Хохшильда алгебры гладких функций на конечномерном вещественном векторном пространстве с коэффициентами в присоединенном представлении.

Ключевые слова: ассоциативная алгебра, бимодуль, кограничный оператор, комплекс, экспоненциальное преобразование.

Когомологии Хохшильда [1] ассоциативных алгебр наряду с когомологиями Шевалле-Эйленберга [2] алгебр Ли играют ведушую роль в теории деформаций различных алгебраических структур [3], [4]. Учитывая неослабеваюший интерес к последней в различных областях математики и физики, включая квантовую теорию [5] (см. также обзор [6]), весьма желательно иметь полностью просчитанные примеры, особенно примеры, важные в приложениях. В предлагаемой работе аналитическими методами вычислены когомологии Хохшильда алгебры гладких функций на конечномерном вешественном пространстве с коэффициентами в присоединенном представлении.

\section{1. КОГОМОЛОГИИ ХОХшИЛЬДА}

Пусть заданы $\mathbb{F}$ - поле характеристики нуль, $\mathfrak{A}$ - ассоциативная алгебра над $\mathbb{F}$ и $\mathfrak{B}$ бимодуль над $\mathfrak{A}$. Будем предполагать, специально этого не оговаривая, что все линейные операции выполняются над $\mathbb{F}$ и все бесконечномерные линейные пространства снабжены естественной локально выпуклой топологией [7]. Все вводимые ниже линейные отображения непрерывные, хотя в явном виде это не проверяется.

Пусть $\otimes \mathfrak{A}=\bigoplus_{q \geqslant 0} \otimes q \mathfrak{A}$ - тензорная алгебра линейного пространства $\mathfrak{A}, \mathcal{C}(\mathfrak{A} ; \mathfrak{B})=$ $\bigoplus_{q \geqslant 0} \mathcal{C}^{q}(\mathfrak{A} ; \mathfrak{B})$ - линейное пространство всех коцепей на $\mathfrak{A}$ со значениями в $\mathfrak{B}$, где $\mathcal{C}^{q}(\mathfrak{A} ; \mathfrak{B})$ суть линейные пространства всех $q$-линейных отображений из $\mathfrak{A}^{q}=\times^{q} \mathfrak{A}$ в $\mathfrak{B}$.

* Математический институт им. В. А. Стеклова РАН, Москва, Россия. E-mail: zharinov@mi.ras.ru 
Кограничный оператор $\delta$ на $\mathcal{C}(\mathfrak{A} ; \mathfrak{B})$ задается правилом

$$
\begin{aligned}
& \delta \omega\left(a_{1}, \ldots, a_{q+1}\right)=a_{1} \omega\left(a_{2}, \ldots, a_{q+1}\right)+ \\
& \quad+\sum_{1 \leqslant r \leqslant q}(-1)^{r} \omega\left(a_{1}, \ldots, a_{r} a_{r+1}, \ldots, a_{q+1}\right)+(-1)^{q+1} \omega\left(a_{1}, \ldots, a_{q}\right) a_{q+1},
\end{aligned}
$$

где $q=0,1, \ldots, \omega \in \mathcal{C}^{q}(\mathfrak{A} ; \mathfrak{B}), a_{1}, \ldots, a_{q+1} \in \mathfrak{A}, \delta \omega \in \mathcal{C}^{q+1}(\mathfrak{A} ; \mathfrak{B})$. Легко проверяется, что $\delta \circ \delta=0$ (здесь и всюду ниже значок о означает композицию отображений), так что определен комплекс $\{\mathcal{C}(\mathfrak{A} ; \mathfrak{B}) ; \delta\}$ с когомологиями

$$
H\{\mathcal{C}(\mathfrak{A} ; \mathfrak{B}) ; \delta\}=H(\mathfrak{A} ; \mathfrak{B})=\bigoplus_{q \geqslant 0} H^{q}(\mathfrak{A} ; \mathfrak{B}) .
$$

Над всякой ассоциативной алгеброй $\mathfrak{A}$ определены два естественных бимодуля:

- тривиальныц, $\mathfrak{B}=\mathbb{F}$, действия $a x=x a=0$ для всех $a \in \mathfrak{A}, x \in \mathbb{F}$;

- присоединенный, $\mathfrak{B}=\mathfrak{A}$, действия $a b, b a$ суть произведения в алгебре $\mathfrak{A}$ для всех $a \in \mathfrak{A}, b \in \mathfrak{B}=\mathfrak{A}$.

В первом случае справедливо

ПРЕДЛОЖЕНИЕ 1.1. Если алгебра $\mathfrak{A}$ унитальная (т.е. содержит единичный элемент), то когомологии

$$
H^{q}(\mathfrak{A} ; \mathbb{F})= \begin{cases}\mathbb{F}, & q=0 \\ 0, & q=1,2, \ldots\end{cases}
$$

ДокаЗАтЕльСтво. Действительно, в этом случае для всех $\omega \in \mathcal{C}^{q}(\mathfrak{A} ; \mathbb{F}), q=0,1, \ldots$, легко проверяется гомотопическая формула

где

$$
(\delta \circ \rho+\rho \circ \delta) \omega= \begin{cases}0, & q=0, \\ \omega, & q=1,2, \ldots,\end{cases}
$$

$$
\begin{aligned}
& \delta \omega\left(a_{1}, \ldots, a_{q+1}\right)=\sum_{1 \leqslant r \leqslant q}(-1)^{r} \omega\left(a_{1}, \ldots, a_{r} a_{r+1}, \ldots, a_{q+1}\right), \\
& \rho \omega\left(a_{1}, \ldots, a_{q-1}\right)=-\omega\left(e, a_{1}, \ldots, a_{q-1}\right)
\end{aligned}
$$

для всех $a_{1}, \ldots, a_{q+1} \in \mathfrak{A}(e-$ единичный элемент алгебры $\mathfrak{A})$.

\section{2. АЛГЕБРА ГЛАДКИХ ФУНКЦИЙ}

Будем использовать технику, развитую в работах [8]. Начиная с этого момента $\mathbb{F}=$ $\mathbb{C}, V$ - вешественное $m$-мерное линейное пространство, $\mathbb{V}=\mathbb{C} \otimes V$-его комплексификация, $V^{*}$ и $\mathbb{V}^{*}$ - их дуальные, $\mathfrak{A}=\mathcal{E}(V)=\mathcal{C}^{\infty}(V ; \mathbb{C})$ - алгебра всех гладких комплексных функций на $V$ с естественной топологией равномерной сходимости на компактах вместе с производными всех порядков.

В случае тривиальных коэффициентов пространства $\mathcal{C}^{q}(\mathcal{E}(V) ; \mathbb{C})=\mathcal{E}\left(V^{q}\right)^{*}$ - линейные пространства, дуальные к пространствам $\mathcal{E}\left(V^{q}\right)$, наделенные сильной топологией, так что $q$-коцепи суть обобщенные функции на $V^{q}=\times^{q} V$ с компактными носителями. Когомологии комплекса $\{\mathcal{C}(\mathcal{E}(V) ; \mathbb{C}) ; \delta\}$ описываются предложением 1.1 , поскольку алгебра $\mathcal{E}(V)$ содержит единичный элемент - функцию $e(x) \equiv 1$. 
Остальная часть статьи посвящена когомологиям алгебры $\mathcal{E}(V)$ с коэффициентами в присоединенном бимодуле $\mathfrak{B}=\mathcal{E}(V)$. Здесь пространства $\mathcal{C}^{q}(\mathcal{E}(V) ; \mathcal{E}(V))$ - линейные пространства всех непрерывных $q$-линейных отображений из $\mathcal{E}(V)^{q}$ в $\mathcal{E}(V)$, наделенные сильной топологией. Справедливо представление $\mathcal{C}^{q}(\mathcal{E}(V) ; \mathcal{E}(V))=\mathcal{E}(V) \widehat{\otimes} \mathcal{E}\left(V^{q}\right)^{*}$, где значок означает замыкание тензорного произведения в подходяшей топологии, которая для нас сейчас не важна [7]. В частности, $q$-коцепи суть $\mathcal{E}(V)$-значные обобшенные функции на $V^{q}$ с компактными носителями.

\section{3. ЭКСПОНЕНЦИАЛЬНОЕ ПРЕОБРАЗОВАНИЕ}

Для всякой пары $\xi \in \mathbb{V}^{*}, x \in V$ определено естественное спаривание $(\xi, x)$ ковектора и вектора. Каждому $\xi \in \mathbb{V}^{*}$ поставим в соответствие функцию $e^{\xi} \in \mathcal{E}(V)$, где $e^{\xi}(x)=$ $e^{(\xi, x)}$ для всех $x \in V$.

Пусть $q=0,1, \ldots$ Обозначим через $\operatorname{Exp}\left(\left(V^{*}\right)^{q}\right)$ линейное пространство всех целых функций экспоненциального типа на $\left(\mathbb{V}^{*}\right)^{q} \simeq \mathbb{C}^{m q}$. Преобразование Лапласа $\omega \mapsto L \omega$ определено формулой

$$
L \omega\left(\xi_{1}, \ldots, \xi_{q}\right)=\omega\left(e^{\xi_{1}}, \ldots, e^{\xi_{q}}\right)
$$

для всех $\omega \in \mathcal{E}\left(V^{q}\right)^{*}$ и $\xi_{1}, \ldots, \xi_{q} \in \mathbb{V}^{*}$. Линейное отображение $L: \mathcal{E}\left(V^{q}\right)^{*} \rightarrow \operatorname{Exp}\left(\left(\mathbb{V}^{*}\right)^{q}\right)$ инъективное, его образ $\Phi^{q}=L\left(\mathcal{E}\left(V^{q}\right)^{*}\right)$ состоит из всех целых функций на $\left(\mathbb{V}^{*}\right)^{q}$, растуших не быстрее линейной экспоненты в вешественных направлениях и не быстрее степенной функции в мнимых. Линейное пространство $\Phi^{q}$ наделяется топологией, индуцированной из $\mathcal{E}\left(V^{q}\right)^{*}$, так что определен изоморфизм линейных топологических пространств $L: \mathcal{E}\left(V^{q}\right)^{*} \simeq \Phi^{q}$ (см., например, [9]). Отождествление $L \equiv \mathrm{id}_{\mathcal{E}(V)} \otimes L$ распространяет преобразование Лапласа на $\mathcal{E}(V) \widehat{\otimes} \mathcal{E}\left(V^{q}\right)^{*}$ и приводит к изоморфизму

$$
L: \mathcal{C}^{q}(\mathcal{E}(V) ; \mathcal{E}(V)) \simeq \mathcal{F}^{q}=\mathcal{E}(V) \widehat{\otimes} \Phi^{q} .
$$

"Подкрученное" преобразование $\Lambda: \mathcal{C}^{q}(\mathcal{E}(V) ; \mathcal{E}(V)) \simeq \mathcal{F}^{q}$ задается правилом

$$
\Lambda \omega\left(x ; \xi_{1}, \ldots, \xi_{q}\right)=e^{-(\xi, x)} \omega\left(x ; e^{\xi_{1}}, \ldots, e^{\xi_{q}}\right)
$$

для всех $\omega \in \mathcal{C}^{q}(\mathcal{E}(V) ; \mathcal{E}(V)), x \in V, \xi_{1}, \ldots, \xi_{q} \in \mathbb{V}^{*}, \xi=\sum_{1 \leqslant r \leqslant q} \xi_{r} \in \mathbb{V}^{*}$ и приводит к изоморфизму

$$
\Lambda: \mathcal{C}(\mathcal{E}(V) ; \mathcal{E}(V)) \simeq \mathcal{F}=\bigoplus_{q \geqslant 0} \mathcal{F}^{q} .
$$

Кограничный оператор $\delta: \mathcal{C}(\mathcal{E}(V) ; \mathcal{E}(V)) \rightarrow \mathcal{C}(\mathcal{E}(V) ; \mathcal{E}(V))$ индуцирует дифференциал $d_{\mathcal{F}}=\Lambda \circ \delta \circ \Lambda^{-1}: \mathcal{F} \rightarrow \mathcal{F}$, действующий по правилу

$$
\begin{aligned}
& d_{\mathcal{F}} f\left(x ; \xi_{1}, \ldots, \xi_{q+1}\right)=f\left(x ; \xi_{2}, \ldots, \xi_{q+1}\right)+ \\
& \quad+\sum_{1 \leqslant r \leqslant q}(-1)^{r} f\left(x ; \xi_{1}, \ldots, \xi_{r}+\xi_{r+1}, \ldots, \xi_{q+1}\right)+(-1)^{q+1} f\left(x ; \xi_{1}, \ldots, \xi_{q}\right)
\end{aligned}
$$

для всех $f \in \mathcal{F}^{q}, x \in V, \xi_{1}, \ldots, \xi_{q+1} \in \mathbb{V}^{*}$. Итак, определены комплекс $\left\{\mathcal{F} ; d_{\mathcal{F}}\right\}$ и изоморфизмы комплексов $\Lambda:\{\mathcal{C}(\mathcal{E}(V) ; \mathcal{E}(V)) ; \delta\} \simeq\left\{\mathcal{F} ; d_{\mathcal{F}}\right\}$ и соответствуюших пространств 
когомологий $\Lambda: H(\mathcal{E}(V) ; \mathcal{E}(V)) \simeq H(\mathcal{F})$. Далее, при вычислении дифференциала $d_{\mathcal{F}} f$ переменная $x \in V$ является параметром, т.е. $d_{\mathcal{F}}=\mathrm{id}_{\mathcal{E}(V)} \otimes d$, где дифференциал $d: \Phi \rightarrow$ $\Phi, \Phi=\bigoplus_{q \geqslant 0} \Phi^{q}$, действует по "суженному" правилу (его явньй вид приведен ниже). Таким образом, определен вспомогательный комплекс $\{\Phi ; d\}$ с пространством когомологий $H(\Phi)$ и справедливо

ПРЕДЛОЖЕНИЕ 3.1. Имеет место изоморфизм

$$
H(\mathcal{E}(V) ; \mathcal{E}(V)) \simeq \mathcal{E}(V) \widehat{\otimes} H(\Phi)
$$

\section{4. ЛИНЕЙНЫЕ ОПЕРАЦИИ НА}

4.1. Мультииндексные обозначения. Пусть $\mathbb{Z}^{+}=\{0,1, \ldots\}, \overline{1, m}=\{1, \ldots, m\}$, где $m=\operatorname{dim} \mathbb{V}$. Положим

$$
\mathbb{Z}_{m}^{+}=\left\{K=\left(k_{1}, \ldots, k_{m}\right) ; k_{\alpha} \in \mathbb{Z}^{+}, \alpha \in \overline{1, m}\right\}
$$

и будем писать $|K|=k_{1}+\cdots+k_{m}, K !=k_{1} ! \ldots k_{m}$ ! для всякого $K=\left(k_{1}, \ldots, k_{m}\right) \in \mathbb{Z}_{m}^{+}$. Для каждого $q \in \mathbb{Z}^{+}$положим

$$
(\overline{1, m})_{q}=\left\{\mathrm{A}=\left(\alpha_{1}, \ldots, \alpha_{q}\right) ; \alpha_{r} \in \overline{1, m}, 1 \leqslant r \leqslant q\right\}
$$

и будем писать $|\mathrm{A}|=q, \mathrm{~A}^{\prime}=\left(\alpha_{q}, \ldots, \alpha_{1}\right)$ для всякого $\mathrm{A}=\left(\alpha_{1}, \ldots, \alpha_{q}\right) \in(\overline{1, m})_{q}$.

Фиксируем в дуальном пространстве $\mathbb{V}^{*}$ систему координат, так что теперь $\mathbb{V}^{*}=$ $\left\{\xi=\left(\xi^{1}, \ldots, \xi^{m}\right) ; \xi^{\alpha} \in \mathbb{C}, \alpha \in \overline{1, m}\right\}$. Для каждого $K \in \mathbb{Z}_{m}^{+}$определены моном $\xi^{K}=$ $\left(\xi^{1}\right)^{k_{1}} \ldots\left(\xi^{m}\right)^{k_{m}} \in \Phi^{1}$ и частная производная $\partial_{\xi^{K}}=\left(\partial_{\xi^{1}}\right)^{k_{1}} \ldots\left(\partial_{\xi^{m}}\right)^{k_{m}}$ порядка $|K|$. В частности, $\xi^{0}=1, \partial_{\xi^{0}}=\mathrm{id}$. Далее, для каждого $\alpha \in \overline{1, m}$ положим $K=(\alpha)=(0, \ldots, 0$, $1,0, \ldots, 0)$ (1 стоит на $\alpha$-м месте), так что $\xi^{\alpha}=\xi^{(\alpha)}, \partial_{\xi^{\alpha}}=\partial_{\xi^{(\alpha)}}$. Аналогичным образом для $\alpha, \beta \in \overline{1, m}$ положим $\xi^{\alpha \beta}=\xi^{(\alpha)+(\beta)}=\xi^{\alpha} \xi^{\beta} \in \Phi^{1}$.

4.2. Операции. На линейном пространстве $\Phi$ определена структура ассоциативной алгебры правилом $\phi \in \Phi^{p}, \quad \psi \in \Phi^{q} \mapsto \phi \otimes \psi \in \Phi^{p+q}$, где $\phi \otimes \psi\left(\xi_{1}, \ldots, \xi_{p+q}\right)=$ $\phi\left(\xi_{1}, \ldots, \xi_{p}\right) \psi\left(\xi_{p+1}, \ldots, \xi_{p+q}\right)$ для всех $\xi_{1}, \ldots, \xi_{p+q} \in \mathbb{V}^{*}, p, q=0,1, \ldots$

Кроме того, на $\Phi$ определена основная операция - дифференциал $d$, действующий по правилу

$$
\begin{aligned}
d \phi\left(\xi_{1}, \ldots, \xi_{q+1}\right)= & \phi\left(\xi_{2}, \ldots, \xi_{q+1}\right)+ \\
& +\sum_{1 \leqslant r \leqslant q}(-1)^{r} \phi\left(\xi_{1}, \ldots, \xi_{r}+\xi_{r+1}, \ldots, \xi_{q+1}\right)+(-1)^{q+1} \phi\left(\xi_{1}, \ldots, \xi_{q}\right)
\end{aligned}
$$

для всех $\phi \in \Phi^{q}, \xi_{1}, \ldots, \xi_{q+1} \in \mathbb{V}^{*}, q=0,1, \ldots$ 
ПримеР 4.1. Пусть $K \in \mathbb{Z}_{m}^{+}$и $\xi^{K} \in \Phi^{1}$, тогда

$$
\left(d \xi^{K}\right)\left(\xi_{1}, \xi_{2}\right)=\xi_{2}^{K}-\left(\xi_{1}+\xi_{2}\right)^{K}+\xi_{1}^{K}
$$

для всех $\xi_{1}, \xi_{2} \in \mathbb{V}^{*}$ и, следовательно, $d \xi^{K}=0$ тогда и только тогда, когда $|K|=1$. В частности, $d \xi^{\alpha \beta}=-\left(\xi^{\alpha} \otimes \xi^{\beta}+\xi^{\beta} \otimes \xi^{\alpha}\right)$ для всех $\alpha, \beta \in \overline{1, m}$.

Легко проверяется

ПРЕДЛОЖЕНИЕ 4.1. Отображение $d: \Phi \rightarrow \Phi$ является косым дифференцированием алгебры $\Phi$, m.e.

$$
d(\phi \otimes \psi)=(d \phi) \otimes \psi+(-1)^{p} \phi \otimes(d \psi)
$$

для всех $\phi \in \Phi^{p}, \quad \psi \in \Phi^{q}, \quad p, q=0,1, \ldots$.

Введем ряд вспомогательных операций. Пусть $K \in \mathbb{Z}_{m}^{+}$.

- Линейное отображение $\partial_{\xi^{K}}: \Phi \rightarrow \Phi$ зададим правилом

$$
\partial_{\xi^{K}} \phi\left(\xi_{1}, \ldots, \xi_{q}\right)=\left.\partial_{\xi^{K}} \phi\left(\xi, \xi_{2}, \ldots, \xi_{q}\right)\right|_{\xi=\xi_{1}}=\partial_{\xi_{1}^{K}} \phi\left(\xi_{1}, \ldots, \xi_{q}\right)
$$

для всех $\phi \in \Phi^{q}, \xi_{1}, \ldots, \xi_{q} \in \mathbb{V}^{*}, q \in \mathbb{Z}^{+}$. В частности, $\partial_{\xi^{0}}=\mathrm{id}_{\Phi}$ - тождественное отображение на $\Phi$.

- Линейное отображение $\iota_{K}: \Phi \rightarrow \Phi$ зададим правилом

$$
\iota_{K} \phi\left(\xi_{1}, \ldots, \xi_{q-1}\right)=\left.\partial_{\xi} \phi\left(\xi, \xi_{1}, \ldots, \xi_{q-1}\right)\right|_{\xi=0}
$$

для всех $\phi \in \Phi^{q}, \xi_{1}, \ldots, \xi_{q-1} \in \mathbb{V}^{*}, q \in \mathbb{Z}^{+}$. В частности, положим $\iota=\iota_{0}$, тогда $\iota_{K}=\iota$ ० $\partial_{\xi^{K}}$.

- Линейное отображение $\lambda^{K}: \Phi \rightarrow \Phi$ зададим правилом

$$
\lambda^{K} \phi\left(\xi_{1}, \ldots, \xi_{q+1}\right)=\left.\xi^{K} \phi\left(\xi_{2}, \ldots, \xi_{q+1}\right)\right|_{\xi=\xi_{1}}
$$

для всех $\phi \in \Phi^{q}, \xi_{1}, \ldots, \xi_{q+1} \in \mathbb{V}^{*}, q \in \mathbb{Z}^{+}$. Другими словами, $\lambda^{K}=\xi^{K} \otimes$. Обозначим $\lambda=\lambda^{0}$.

• Положим

$$
\tau_{K}=\frac{1}{K !} \lambda^{K} \circ \iota_{K}, \quad T_{p}=\sum_{|K| \leqslant p} \tau_{K}, \quad \rho_{p}=\mathrm{id}_{\Phi}-T_{p}, \quad p \in \mathbb{Z}^{+},
$$

так что $\tau_{K} \phi$ - моном мультистепени $K, T_{p} \phi$ - многочлен степени $p, \rho_{p} \phi$ - соответствующий остаток разложения в ряд Тейлора функции $\phi \in \Phi^{q}$ по первому аргументу. Обозначим $\tau=\tau_{0}, \rho=\rho_{0}$.

ПРЕДЛОЖЕНИЕ 4.2. Справедливы следующие равенства:

1. $d \circ \iota+\iota \circ d=\tau$;

2. $d \circ \lambda+\lambda \circ d=\lambda \circ \lambda$;

3. $\iota \circ \lambda=\mathrm{id}_{\Phi}, \lambda \circ \iota=\tau, \iota \circ \lambda-\lambda \circ \iota=\rho$;

4. $d \circ \tau-\tau \circ d=0, d \circ \rho-\rho \circ d=0$. 
ПРЕДЛОЖЕНИЕ 4.3. Для любого $K \in \mathbb{Z}_{m}^{+}, \quad K \neq 0$, справедливы равенства

1. $d \circ \partial_{\xi^{K}}-\partial_{\xi^{K}} \circ d=\lambda \circ \partial_{\xi^{K}}$;

2. $d \circ \iota_{K}+\iota_{K} \circ d=-\rho \circ \partial_{\xi^{K}}=-\partial_{\xi^{K}} \circ \rho_{|K|}$;

3. $d \circ \lambda^{K}+\lambda^{K} \circ d=d\left(\lambda^{K}\right)=\left(d \xi^{K}\right) \otimes$, причем $d\left(\lambda^{K}\right)=0$ тогда и только тогда, $\kappa о г \partial a|K|=1$;

4. $\partial_{\xi^{K}} \circ \lambda^{L}=L ! \lambda^{L-K} /(L-K) !, \quad \iota_{K} \circ \lambda^{L}=L ! \delta_{K}^{L} \mathrm{id}_{\Phi}, \quad L \in \mathbb{Z}_{m}^{+}, \quad \delta_{K}^{L}-$ cимвол Кронекера.

Доказательство обоих предложений проводится последовательно прямыми вычислениями.

Нам в основном понадобится следуюший частньй случай.

ПРЕДЛОЖЕНИЕ 4.4. Для любъх $\alpha, \beta \in \overline{1, m}$ справедливы равенства:

1. $d \circ \partial_{\xi^{\alpha}}-\partial_{\xi^{\alpha}} \circ d=\lambda \circ \partial_{\xi^{\alpha}}=\partial_{\xi^{\alpha}} \circ\left(\sum_{1 \leqslant \gamma \leqslant m} \lambda^{\gamma} \circ \partial_{\xi^{\gamma}}\right)$;

2. $d \circ \iota_{\alpha}+\iota_{\alpha} \circ d=-\partial_{\xi \alpha} \circ \rho_{1}$;

3. $d \circ \lambda^{\alpha}+\lambda^{\alpha} \circ d=0$;

4. $\partial_{\xi^{\alpha}} \circ \lambda^{\beta}=\delta_{\alpha}^{\beta} \lambda, \iota_{\alpha} \circ \lambda^{\beta}=\delta_{\alpha}^{\beta} \operatorname{id}_{\Phi}$.

4.3. Симметрическая группа. Напомним элементарные свойства симметрической группы $\mathfrak{S}_{q}, q \in \mathbb{N}=\{1,2, \ldots\}$, т.е. группы подстановок множества $\overline{1, q}=\{1, \ldots, q\}$, так что $\{1, \ldots, q\} \mapsto\{\pi(1), \ldots, \pi(q)\}$ для каждого $\pi \in \mathfrak{S}_{q}$.

1. Определен морфизм групп sign: $\mathfrak{S}_{q} \rightarrow\{ \pm 1\}, \pi \mapsto \operatorname{sign} \pi-$ знак подстановки $\pi$.

2. Подстановка $\varepsilon$ называется элементарной, если она меняет местами два соседних индекса, а остальные оставляет на своих местах. Для всякой подстановки $\pi \in \mathfrak{S}_{q}$ сушествуют элементарные подстановки $\varepsilon_{1}, \ldots, \varepsilon_{n} \in \mathfrak{S}_{q}, n=n(\pi)$, такие, что $\pi=\varepsilon_{1} \circ \cdots \circ \varepsilon_{n}$, $\operatorname{sign} \pi=(-1)^{n}$

3. Определено действие группы $\mathfrak{S}_{q}$ на множестве $(\overline{1, m})_{q}, \mathfrak{S}_{q} \ni \pi:(\overline{1, m})_{q} \rightarrow(\overline{1, m})_{q}$ правилом $\mathrm{A}=\left(\alpha_{1}, \ldots, \alpha_{q}\right) \mapsto \pi \mathrm{A}=\left(\alpha_{\pi(1)}, \ldots, \alpha_{\pi(q)}\right)$.

4. Пусть $\mathcal{K}$ - вешественное или комплексное линейное пространство. Обозначим $\mathcal{K}^{(\overline{1, m})_{q}}=\left\{\phi=\left(\phi_{\mathrm{A}}\right):(\overline{1, m})_{q} \rightarrow \mathcal{K}\right\}$ множество отображений из $(\overline{1, m})_{q}$ в $\mathcal{K}$. Определено действие группы $\mathfrak{S}_{q}$ на $\mathcal{K}^{(\overline{1, m})_{q}}$ правилом $\mathfrak{S}_{q} \ni \pi: \mathcal{K}^{(\overline{1, m})_{q}} \rightarrow \mathcal{K}^{(\overline{1, m})_{q}}, \phi \mapsto \pi \phi$, где $(\pi \phi)_{\mathrm{A}}=\phi_{\pi \mathrm{A}}$.

5. Определена операция кососимметризации $\mathfrak{a}: \mathcal{K}^{(\overline{1, m})_{q}} \rightarrow \mathcal{K}^{(\overline{1, m})_{q}}$ правилом $\phi \mapsto$ $\mathfrak{a} \phi=(1 / q !) \sum_{\pi \in \mathfrak{S}_{q}} \operatorname{sign} \pi \cdot \pi \phi$. Будем писать $(\mathfrak{a} \phi)_{\mathrm{A}}=\phi_{[\mathrm{A}]}=\phi_{\left[\alpha_{1} \ldots \alpha_{q}\right]}$, где квадратные скобки означают кососимметризацию по находяшимся внутри индексам. Легко проверяются следующие свойства кососимметризации:

- $\mathfrak{a} \circ \mathfrak{a}=\mathfrak{a}$, т.е. $\mathfrak{a}-$ проектор;

- $\mathfrak{a}(\pi \phi)=\operatorname{sign} \pi \cdot \mathfrak{a} \phi$ для всех $\phi \in \mathcal{K}^{(\overline{1, m})_{q}}$ и $\pi \in \mathfrak{S}_{q}$.

ЛЕмма 4.1. Для любъх $\phi \in \mathcal{K}^{(\overline{1, m})_{q}} u \pi \in \mathfrak{S}_{q}$ существуют $\phi_{1}, \ldots, \phi_{n} \in \mathcal{K}^{(\overline{1, m})_{q}}$ $u \varepsilon_{1}, \ldots, \varepsilon_{n} \in \mathfrak{S}_{q}, \varepsilon_{r} \phi_{r}=\phi_{r}, 1 \leqslant r \leqslant n$, maкue, ито $\phi-\operatorname{sign} \pi \cdot \pi \phi=\sum_{1 \leqslant r \leqslant n} \phi_{r}$.

ДокАЗАТЕЛЬСТво. Пусть $\pi=\varepsilon_{1} \circ \cdots \circ \varepsilon_{n}, \operatorname{sign} \pi=(-1)^{n}$. Тогда годятся $\phi_{r}=$ $(-1)^{n-r}\left(\varepsilon_{r}+\mathrm{id}\right)\left(\varepsilon_{r+1} \ldots \varepsilon_{n} \phi\right), 1 \leqslant r \leqslant n-1, \phi_{n}=\left(\varepsilon_{n}+\mathrm{id}\right) \phi$, где id - тождественное отображение. 
Лемма 4.2. Для любого $\phi \in \mathcal{K}^{(\overline{1, m})_{q}}$ cуществуют такие $\phi_{1}, \ldots, \phi_{N} \in \mathcal{K}^{(\overline{1, m})_{q}} u$ $\varepsilon_{1}, \ldots, \varepsilon_{N} \in \mathfrak{S}_{q}, \quad \varepsilon_{r} \phi_{r}=\phi_{r}, \quad 1 \leqslant r \leqslant N$, чmo $\phi-\mathfrak{a} \phi=\sum_{1 \leqslant r \leqslant N} \phi_{r}$.

ДОКАЗАТЕЛЬСтво. Заметим, что $\phi-\mathfrak{a} \phi=(1 / q !) \sum_{\pi \in \mathfrak{S}_{q}}(\phi-\operatorname{sign} \pi \cdot \pi \phi)$ и воспользуемся предыдушей леммой.

\section{5. ВЫЧИСЛЕНИЕ КОГОМОЛОГИЙ}

5.1. Подготовительные леммы. Введем ряд обозначений. Для $\phi, \psi \in \Phi^{q}, q \in$ $\mathbb{Z}^{+}$, будем писать $\phi \sim \psi$, если $\phi-\psi=d \chi$ для некоторого $\chi \in \Phi^{q-1}$. Далее, для мультииндекса $\mathrm{A}=\left(\alpha_{1}, \ldots, \alpha_{q}\right) \in(\overline{1, m})_{q}, q \in \mathbb{Z}^{+}$, положим $(\otimes \xi)^{\mathrm{A}}=\xi^{\alpha_{1}} \otimes \cdots \otimes \xi^{\alpha_{q}} \in \Phi^{q}$ и $(\circ \iota)_{\mathrm{A}}=\iota_{\alpha_{1}} \circ \cdots \circ \iota_{\alpha_{q}}$. Напомним, что $\mathrm{A}^{\prime}=\left(\alpha_{q}, \ldots, \alpha_{1}\right)$.

Лемма 5.1. Пусть $\phi=\sum_{|\mathrm{A}|=p}(\otimes \xi)^{\mathrm{A}} \otimes \psi_{\mathrm{A}^{\prime}} \in \Phi^{q}, \quad \psi_{\mathrm{A}} \in \Phi^{q-p}, \quad p \leqslant q . \quad$ Тогдa $\psi_{\mathrm{A}}=(\circ \iota)_{\mathrm{A}} \phi$. Если, кроме того, $\varepsilon \psi=\psi$, где әлементарная подстановка в меняет местами индексы $r$ и $r+1,1 \leqslant r<p$, то $\phi \sim \sum_{|\mathrm{A}|=r-1}(\otimes \xi)^{\mathrm{A}} \otimes \chi_{\mathrm{A}^{\prime}}$, где $\chi_{\mathrm{A}} \in$ $\Phi^{q-r+1}, T_{1} \chi_{\mathrm{A}}=0$.

ДоКАЗАТЕЛЬСтво. Достаточно воспользоваться предложением 4.1 и примером 4.1.

Лемма 5.2. Пусть $\phi \in \Phi^{q}, d \phi=0, q \in \mathbb{Z}^{+}$. Тогда

$$
\phi \sim \sum_{|\mathrm{A}|=q}(\otimes \xi)^{\mathrm{A}} \cdot c_{\mathrm{A}^{\prime}}+\sum_{|\mathrm{B}| \leqslant q-1}(\otimes \xi)^{\mathrm{B}} \otimes \psi_{\mathrm{B}^{\prime}}
$$

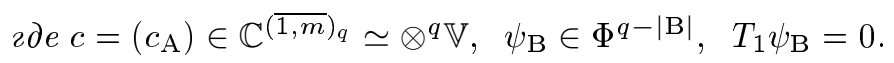

ДокАЗАТЕЛЬСТво. Воспользуемся методом индукции. По условию $\phi \in \Phi^{q}, d \phi=0$. С помошью первого равенства из предложения 4.2 получаем

$$
\phi=\tau \phi+\rho \phi=d(\iota \phi)+\sum_{1 \leqslant \alpha \leqslant m} \xi^{\alpha} \otimes \phi_{\alpha}+\psi \sim \sum_{|\mathrm{A}|=1}(\otimes \xi)^{\mathrm{A}} \otimes \phi_{\mathrm{A}^{\prime}}+\psi,
$$

где $\psi=\rho_{1} \phi$, так что $T_{1} \psi=0$. Предположим теперь, что для некоторого $r<q$ справедливо представление

$$
\phi \sim \tilde{\phi}=\sum_{|\mathrm{A}|=r}(\otimes \xi)^{\mathrm{A}} \otimes \phi_{\mathrm{A}^{\prime}}+\sum_{|\mathrm{B}| \leqslant r-1}(\otimes \xi)^{\mathrm{B}} \otimes \psi_{\mathrm{B}^{\prime}}
$$

где $\phi_{\mathrm{A}} \in \Phi^{q-r}, \psi_{\mathrm{B}} \in \Phi^{q-|\mathrm{B}|}, T_{1} \psi_{\mathrm{B}}=0$, и докажем, что такое же представление справедливо и для $r+1$. С помошью предложения 4.1 и примера 4.1 получаем

$$
d \tilde{\phi}=0=(-1)^{r} \sum_{|\mathrm{A}|=r}(\otimes \xi)^{\mathrm{A}} \otimes d \phi_{\mathrm{A}^{\prime}}+\sum_{|\mathrm{B}| \leqslant r-1}(-1)^{|\mathrm{B}|}(\otimes \xi)^{\mathrm{B}} \otimes d \psi_{\mathrm{B}^{\prime}}
$$

Пусть $\mathrm{A}=\left(\alpha_{1}, \ldots, \alpha_{r}\right) \in(\overline{1, m})_{r}$. Действуя на предыдушее равенство оператором $(\circ \iota)_{\mathrm{A}^{\prime}}$, с помощью леммы 5.1 получаем

$$
(-1)^{r} d \phi_{\mathrm{A}^{\prime}}+\sum_{0 \leqslant p \leqslant r-1}(-1)^{p} \iota_{\alpha_{r}} \ldots \iota_{\alpha_{p+1}} d \psi_{\alpha_{p} \ldots \alpha_{1}}=0
$$


или, в силу второго равенства из предложения 4.4,

$$
(-1)^{r}\left(d \phi_{\mathrm{A}^{\prime}}+\partial_{\xi^{\alpha}} \psi_{\alpha_{r-1} \ldots \alpha_{1}}\right)+\sum_{0 \leqslant p \leqslant r-2}(-1)^{p+1} \iota_{\alpha_{r}} \ldots \iota_{\alpha_{p+3}} \iota_{\alpha_{p+2} \alpha_{p+1}} \psi_{\alpha_{p} \ldots \alpha_{1}}=0
$$

где $\iota_{\alpha \beta}=\iota_{\alpha} \circ \partial_{\xi^{\beta}}=\iota \circ \partial_{\xi^{\alpha}} \circ \partial_{\xi^{\beta}}=\iota_{\beta \alpha}$ и было учтено, что $\rho_{1} \psi_{\mathrm{B}}=\psi_{B}$, поскольку $T_{1} \psi_{\mathrm{B}}=0$. Действуя на это равенство оператором $\iota$, с помошью первого равенства из предложения 4.2 получаем

$$
\tau \phi_{\mathrm{A}^{\prime}}=\sum_{0 \leqslant p \leqslant r-2}(-1)^{r-p} \iota_{\alpha_{r}} \ldots \iota_{\alpha_{p+3}} \iota_{\alpha_{p+2} \alpha_{p+1}} \psi_{\alpha_{p} \ldots \alpha_{1}}
$$

где учтено, что $\iota_{\alpha} \psi_{\mathrm{B}}=0$. Таким образом,

$$
\phi_{\mathrm{A}^{\prime}}=\tau \phi_{\mathrm{A}^{\prime}}+\sum_{1 \leqslant \alpha \leqslant m} \xi^{\alpha} \otimes \phi_{\alpha \mathrm{A}^{\prime}}+\rho_{1} \phi_{\mathrm{A}^{\prime}}=\sum_{1 \leqslant \alpha \leqslant m} \xi^{\alpha} \otimes \phi_{\alpha \mathrm{A}^{\prime}}+\tilde{\psi}_{\mathrm{A}^{\prime}}+\sum_{0 \leqslant p \leqslant r-2} \omega_{\mathrm{A}^{\prime}}^{(p)},
$$

где $\tilde{\psi}_{\mathrm{A}^{\prime}}=\rho_{1} \phi_{\mathrm{A}^{\prime}}, \quad T_{1} \tilde{\psi}_{\mathrm{A}^{\prime}}=0, \quad \omega_{\mathrm{A}^{\prime}}^{(p)}=(-1)^{r-p} \iota_{\alpha_{r}} \ldots \iota_{\alpha_{p+3}} \iota_{\alpha_{p+2} \alpha_{p+1}} \psi_{\alpha_{p} \ldots \alpha_{1}}$, причем $\varepsilon_{p} \omega^{(p)}=\omega^{(p)}$, элементарная подстановка $\varepsilon_{p}$ меняет местами индексы $p+1$ и $p+2$. Подставив последнее выражение в исходное представление, получим

$$
\begin{aligned}
\tilde{\phi} & =\sum_{|\mathrm{A}|=r} \sum_{1 \leqslant \alpha \leqslant m}(\otimes \xi)^{\mathrm{A}} \otimes \xi^{\alpha} \otimes \phi_{\alpha \mathrm{A}^{\prime}}+\sum_{|\mathrm{A}|=r}(\otimes \xi)^{\mathrm{A}} \otimes \tilde{\psi}_{\mathrm{A}^{\prime}}+ \\
& +\sum_{0 \leqslant p \leqslant r-2} \sum_{|\mathrm{A}|=r}(\otimes \xi)^{\mathrm{A}} \otimes \omega_{\mathrm{A}^{\prime}}^{(p)}+\sum_{|\mathrm{B}| \leqslant r-1}(\otimes \xi)^{\mathrm{B}} \otimes \psi_{\mathrm{B}^{\prime}} \sim \\
\sim & \sum_{|\mathrm{A}|=r+1}(\otimes \xi)^{\mathrm{A}} \otimes \tilde{\phi}_{\mathrm{A}^{\prime}}+\sum_{|\mathrm{B}| \leqslant r}(\otimes \xi)^{\mathrm{B}} \otimes \tilde{\psi}_{\mathrm{B}^{\prime}},
\end{aligned}
$$

где $\tilde{\phi}_{\alpha_{r+1} \ldots \alpha_{1}}=\phi_{\alpha_{r+1} \mathrm{~A}^{\prime}}, \mathrm{A}^{\prime}=\left(\alpha_{r} \ldots \alpha_{1}\right), \tilde{\psi}_{\mathrm{B}}=\psi_{\mathrm{B}}$ при $|\mathrm{B}|=r-1, \tilde{\psi}_{\mathrm{B}}=\psi_{\mathrm{B}}+\chi_{\mathrm{B}}^{(|\mathrm{B}|)}$ при $0 \leqslant|\mathrm{~B}| \leqslant r-2$ (см. лемму 5.1). Итак, требуемое представление для $r+1$ доказано. Полагая теперь $r=q$ и учитывая, что $\Phi^{0}=\mathbb{C}$, завершаем доказательство леммы.

Лемма 5.3. Для всякого $c=\left(c_{\mathrm{A}}\right) \in \otimes^{q} \mathbb{V}$ справедливо утвержсдение

$$
\phi=\sum_{|\mathrm{A}|=q}(\otimes \xi)^{\mathrm{A}} \cdot c_{\mathrm{A}^{\prime}} \sim \mathfrak{a} \phi=\sum_{|\mathrm{A}|=q}(\wedge \xi)^{\mathrm{A}} \cdot v_{\mathrm{A}^{\prime}}
$$

где значок $\wedge$ означает внешнее (кососимметризованное) произведение, $v=\left(v_{\mathrm{A}}\right)=$ $\mathfrak{a} c \in \wedge^{q} \mathbb{V}-q$-вектор.

ДокАЗАТЕЛЬСтво. Следует воспользоваться леммой 4.2 и модифицировать доказательство леммы 5.1.

Лемма 5.4. Для любых $\psi_{\mathrm{B}} \in \Phi^{q-|\mathrm{B}|}, \quad T_{1} \psi_{\mathrm{B}}=0,|\mathrm{~B}| \leqslant q-1$, справедливо утверждение

$$
\phi=\sum_{|\mathrm{B}| \leqslant q-1}(\otimes \xi)^{\mathrm{B}} \otimes \psi_{\mathrm{B}^{\prime}} \sim \tilde{\phi}=\sum_{|\mathrm{B}| \leqslant q-1}(\wedge \xi)^{\mathrm{B}} \otimes \tilde{\psi}_{\mathrm{B}^{\prime}},
$$

əде $\tilde{\psi}_{\mathrm{B}} \in \Phi^{q-|\mathrm{B}|}, \quad T_{1} \tilde{\psi}_{\mathrm{B}}=0, \quad(\mathfrak{a} \tilde{\psi})_{\mathrm{B}}=\tilde{\psi}_{\mathrm{B}}, \quad|\mathrm{B}| \leqslant q-1, \quad \tilde{\psi}_{\mathrm{B}}=(\mathfrak{a} \psi)_{\mathrm{B}} n p u|\mathrm{~B}|=q-1$. 
ДокАЗАтЕЛЬСтво. Пусть сначала $|\mathrm{B}|=q-1$. Лемма 4.2 дает

$$
\sum_{|\mathrm{B}|=q-1}(\otimes \xi)^{\mathrm{B}} \otimes \psi_{\mathrm{B}^{\prime}}=\sum_{|\mathrm{B}|=q-1}(\otimes \xi)^{\mathrm{B}} \otimes\left((\mathfrak{a} \psi)_{\mathrm{B}^{\prime}}+\sum_{1 \leqslant r \leqslant N} \psi_{r \mathrm{~B}^{\prime}}\right),
$$

где $\psi_{r \mathrm{~B}} \in \Phi^{q-|\mathrm{B}|}, T_{1} \psi_{r \mathrm{~B}}=0,\left(\varepsilon_{r} \psi_{r}\right)_{\mathrm{B}}=\psi_{r \mathrm{~B}}$ для некоторых элементарных подстановок $\varepsilon_{r}, 1 \leqslant r \leqslant N$. Согласно лемме 5.1

$$
\sum_{|\mathrm{B}|=q-1}(\otimes \xi)^{\mathrm{B}} \otimes \psi_{r \mathrm{~B}^{\prime}} \sim \sum_{|\mathrm{B}|=s-1}(\otimes \xi)^{\mathrm{B}} \otimes \chi_{r \mathrm{~B}^{\prime}}
$$

где $s=s(r)<q-1, \chi_{r \mathrm{~B}} \in \Phi^{q-|\mathrm{B}|}, T_{1} \chi_{r \mathrm{~B}}=0$, откуда

$$
\phi \sim \sum_{|\mathrm{B}|=q-1}(\wedge \xi)^{\mathrm{B}} \otimes \tilde{\psi}_{\mathrm{B}^{\prime}}+\sum_{|\mathrm{B}| \leqslant q-2}(\otimes \xi)^{\mathrm{B}} \otimes \psi_{\mathrm{B}^{\prime}}^{\prime},
$$

где $\tilde{\psi}=\mathfrak{a} \psi, \psi_{\mathrm{B}}^{\prime} \in \Phi^{q-\mid \mathrm{B}}, T_{1} \psi_{\mathrm{B}}^{\prime}=0$. Повторяя эти рассуждения для $|\mathrm{B}|=q-2, q-$ $3, \ldots$, завершаем доказательство леммы.

Нам понадобится лемма Пуанкаре, которую мы сформулируем в удобной для нас форме.

Лемма 5.5. Пусть $\phi \in\left(\Phi^{p}\right)^{(\overline{1, m})_{q}}, T_{r} \phi=0, p, q, r \in \mathbb{Z}^{+}$. Ecлu $\mathfrak{a} \phi=\phi, \mathfrak{a}\left(\partial_{\xi} \phi\right)=0$, то $\phi=0$ при $q=0$, а при $q>0$ найдется $\omega \in\left(\Phi^{p}\right)^{(\overline{1, m})_{q-1}}, \quad T_{r+1} \omega=0, \quad \mathfrak{a} \omega=\omega$, mакое, что $\phi=\mathfrak{a}\left(\partial_{\xi} \omega\right)$.

ДокАЗАТЕльСТво. Следует обратить внимание на то, что частные производные $\partial_{\xi^{\alpha}}$, $\alpha \in \overline{1, m}$, берутся только по первому аргументу $\xi_{1} \in \mathbb{C}^{m}$ функции $\phi_{\mathrm{A}}\left(\xi_{1}, \ldots, \xi_{p}\right), \mathrm{A}=$ $\left(\alpha_{1}, \ldots, \alpha_{q}\right)$, и воспользоваться стандартным выражением для гомотопической формулы Пуанкаре.

Лемма 5.6. Пусть

$$
\phi=\sum_{|\mathrm{B}| \leqslant q-1}(\wedge \xi)^{\mathrm{B}} \otimes \psi_{\mathrm{B}^{\prime}}
$$

əде $\psi_{\mathrm{B}} \in \Phi^{q-|\mathrm{B}|}, T_{1} \psi_{\mathrm{B}}=0$, причем $d \phi=0$. Тогда $\phi \sim 0$.

ДОКАЗАТЕЛЬСтво. Для удобства положим $\psi_{\mathrm{B}}^{(p)}=\psi_{\mathrm{B}}$ для всех $|\mathrm{B}|=p, 0 \leqslant p \leqslant$ $q-1$. Тогда условие $d \phi=0$ запишется в виде

$$
d \phi=\sum_{0 \leqslant p \leqslant q-1}(-1)^{p} \sum_{|\mathrm{B}|=p}(\wedge \xi)^{\mathrm{B}} \otimes d \psi_{\mathrm{B}^{\prime}}^{(p)}=0 .
$$

Действуя на это равенство оператором $(\circ \iota)_{\mathrm{A}^{\prime}}, \mathrm{A}=\left(\alpha_{1}, \ldots, \alpha_{q}\right)$, получаем

$$
(-1)^{q} \partial_{\xi^{\alpha}} \psi_{\alpha_{q-1} \ldots \alpha_{1}}^{(q-1)}+\sum_{0 \leqslant p \leqslant q-2}(-1)^{p+1} \iota_{\alpha_{q}} \ldots \iota_{\alpha_{p+3}} \iota_{\alpha_{p+2} \alpha_{p+1}} \psi_{\alpha_{p} \ldots \alpha_{1}}^{(p)}=0,
$$


где учтено, что в силу второго равенства из предложения $4.4 \iota_{\alpha} d \psi=-\partial_{\xi} \alpha$, если $T_{1} \psi=0$, и что $\iota_{\alpha} \circ \partial_{\xi^{\beta}}=\iota_{\alpha \beta}$. Кососимметризуя это равенство, получим $\mathfrak{a}\left(\partial_{\xi} \psi^{(q-1)}\right)=0$, поскольку $\iota_{\alpha \beta}=\iota_{\beta \alpha}$. Отсюда в силу леммы 5.5 имеем $\psi_{\mathrm{B}}^{(q-1)}=\mathfrak{a}\left(\partial_{\xi} \omega^{(1)}\right)_{\mathrm{B}}$, где $\omega^{(1)} \in$ $\left(\Phi^{1}\right)^{(\overline{1, m})_{q-1}}, T_{2} \omega_{\mathrm{B}}^{(1)}=0$. Подставляя это представление в выражение для $d \phi=0$ и действуя оператором $(\circ \iota)_{\mathrm{A}^{\prime}}$, где теперь $\mathrm{A}=\left(\alpha_{1}, \ldots, \alpha_{q-1}\right)$, получим

$$
(-1)^{q-1} d \mathfrak{a}\left(\partial_{\xi} \omega^{(1)}\right)_{\mathrm{A}^{\prime}}+(-1)^{q-2+1} \partial_{\xi^{\alpha_{q-1}}} \psi_{\alpha_{q-2} \ldots \alpha_{1}}^{(q-2)}+\cdots
$$

где опущенные члены содержат операторы вида $\iota_{\alpha \beta}$. Кососимметризуя, получим

$$
d \mathfrak{a}\left(\partial_{\xi} \omega^{(1)}\right)_{\mathrm{A}^{\prime}}+\mathfrak{a}\left(\partial_{\xi} \psi^{(q-2)}\right)_{\mathrm{A}^{\prime}}=0
$$

и первое равенство из предложения 4.4 дает

$$
\mathfrak{a}\left[\partial_{\xi}\left(d \omega^{(1)}+\sum_{1 \leqslant \beta \leqslant m} \xi^{\beta} \otimes \partial_{\xi^{\beta}} \omega^{(1)}+\psi^{(q-2)}\right)\right]_{\mathrm{A}^{\prime}}=0
$$

откуда в силу леммы 5.5

$$
\psi_{\mathrm{B}}^{(q-2)}=-d \omega_{\mathrm{B}}^{(1)}-\sum_{1 \leqslant \beta \leqslant m} \xi^{\beta} \otimes \partial_{\xi^{\beta}} \omega_{\mathrm{B}}^{(1)}+\mathfrak{a}\left(\partial_{\xi} \omega^{(2)}\right)_{\mathrm{B}},
$$

где $\omega^{(2)} \in\left(\Phi^{2}\right)^{(\overline{1, m})_{q-2}}, T_{2} \omega_{\mathrm{B}}^{(2)}=0,|\mathrm{~B}|=q-2$.

Исходное выражение для $\phi$ теперь принимает вид

$$
\begin{aligned}
\phi & =\sum_{|\mathrm{B}|=q-1}(\wedge \xi)^{\mathrm{B}} \otimes \mathfrak{a}\left(\partial_{\xi} \omega^{(1)}\right)_{\mathrm{B}^{\prime}}+ \\
& +\sum_{|\mathrm{B}|=q-2}(\wedge \xi)^{\mathrm{B}} \otimes\left(-d \omega_{\mathrm{B}^{\prime}}^{(1)}-\sum_{1 \leqslant \beta \leqslant m} \xi^{\beta} \otimes \partial_{\xi^{\beta}} \omega_{\mathrm{B}^{\prime}}^{(1)}+\mathfrak{a}\left(\partial_{\xi} \omega^{(2)}\right)_{\mathrm{B}^{\prime}}\right)+ \\
& +\sum_{|\mathrm{B}| \leqslant q-3}(\wedge \xi)^{\mathrm{B}} \otimes \psi_{\mathrm{B}^{\prime}}^{(|\mathrm{B}|)} \sim \\
\sim & \sum_{|\mathrm{B}|=q-2}(\wedge \xi)^{\mathrm{B}} \otimes \mathfrak{a}\left(\partial_{\xi} \omega^{(2)}\right)_{\mathrm{B}^{\prime}}+\sum_{|\mathrm{B}| \leqslant q-3}(\wedge \xi)^{\mathrm{B}} \otimes \tilde{\psi}_{\mathrm{B}^{\prime}}^{(|\mathrm{B}|)}
\end{aligned}
$$

с учетом леммы 5.4. Повторяя эти рассуждения для $|\mathrm{B}|=q-2, q-3, \ldots$, завершаем доказательство леммы.

5.2. Когомологии вспомогательного комплекса. Теперь мы в состоянии вычислить пространство когомологий $H(\Phi)$ вспомогательного комплекса $\{\Phi ; d\}$.

ТеОРема 1. Справедливы равенства

$$
H^{q}(\Phi)=\wedge^{q} \mathbb{V}, \quad q \in \mathbb{Z}^{+},
$$

где $\wedge^{q} \mathbb{V}$ есть q-я внешняя степень комплексного векторного пространства $\mathbb{V}$ (в частности, $\left.\wedge^{0} \mathbb{V}=\mathbb{C}\right)$. 
ДокАЗАТЕльСТво. Достаточно последовательно воспользоваться леммами 5.2-5.4, 5.6 и убедиться, что для любого $v \in \wedge^{q} \mathbb{V}$ функция $\phi=\sum_{|\mathrm{A}|=q}(\wedge \xi)^{\mathrm{A}} \cdot v_{\mathrm{A}^{\prime}}$ удовлетворяет равенству $d \phi=0$, причем $\phi=d \psi, \psi \in \Phi^{q-1}$ лишь в случае $v=0, \psi=0$.

5.3. Когомологии алгебры гладких функций. Объединяя доказанную теорему с предложением 3.1 , убеждаемся в справедливости основного результата данной работы.

Теорема 2. Имеет место изоморфизм

$$
H(\mathcal{E}(V) ; \mathcal{E}(V)) \simeq \mathcal{E}(V) \otimes(\wedge \mathbb{V})
$$

где $\wedge \mathbb{V}$ - внешняя алгебра комплексного векторного пространства $\mathbb{V}$.

Заметим, что значок`замыкания тензорного произведения здесь не нужен, поскольку линейное пространство $\wedge \mathbb{V}$ конечномерно, как и исходное вешественное пространство $V$.

\section{6. ОБСУЖДЕНИЕ}

Большинство работ в данной области посвящено более общей ситуации, когда исходная алгебра $\mathcal{E}(M)$ - алгебра всех гладких функций на гладком вещественном многообразии $M$. При этом рассматриваются различные классы коцепей на $\mathcal{E}(M)$, из которых основные два: непрерывные и дифференциальные. Подробнее, линейное пространство $\mathcal{E}(M)=\mathcal{C}^{\infty}(M ; \mathbb{C})\left(\right.$ или $\left.\mathcal{C}^{\infty}(M ; \mathbb{R})\right)$ наделяется естественной топологией равномерной сходимости на компактах вместе с производными всех порядков. Пространства непрерывны $x$ коцепей $\mathcal{C}^{q}(\mathcal{E}(M) ; \mathcal{E}(M))$ суть линейные пространства всех непрерывных $q$-линейных отображений из $\mathcal{E}(M)^{q}$ в $\mathcal{E}(M)$, наделенные сильной топологией, а прос-

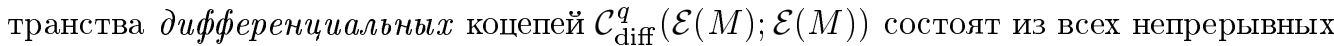
коцепей, задаваемых дифференциальными операторами конечного порядка по каждому аргументу. Полное описание непрерывных и дифференциальных когомологий, судя по всему, пока отсутствует, имеются лишь частные результаты, в основном для $q \leqslant 3$ (см., например, [10]), поскольку когомологии именно этих порядков используются в теории деформаций. В любом случае прямое аналитическое доказательство одного из основных ингредиентов теории деформаций представляется весьма полезным.

В изученном нами случае $M=V$ - векторное пространство, переход к дифференциальным когомологиям сводится к замене вспомогательного комплекса $\{\Phi ; d\}$ на комплекс $\left\{\Phi_{\text {pol }} ; d\right\}$, где пространства $\Phi_{\text {pol }}^{q}=\mathbb{C}\left[\xi_{1}, \ldots, \xi_{q}\right] \subset \Phi^{q}$ суть пространства многочленов векторных переменных $\xi_{1}, \ldots, \xi_{q} \in \mathbb{C}^{m}, q \in \mathbb{Z}^{+}$, а дифференциал $d: \Phi_{\mathrm{pol}} \rightarrow \Phi_{\mathrm{pol}}$ есть сужение исходного дифференциала $d$ с $\Phi$ на $\Phi_{\text {роl }}$. Легко проверяется, что все введенные выше операции и вспомогательные результаты допускают сужение на $\Phi_{\text {pol }}$, так что справедливо

ПРЕДЛОЖЕНИЕ 6.1. Имеют место равенства

$$
H^{q}\left(\Phi_{\mathrm{pol}}\right)=\wedge^{q} \mathbb{V}=H^{q}(\Phi), \quad q \in \mathbb{Z}^{+} .
$$

В свою очередь, отсюда вытекает 
ПРЕДЛОЖЕНИЕ 6.2. Имеет место изоморфизм

$$
H_{\text {diff }}(\mathcal{E}(V) ; \mathcal{E}(V)) \simeq \mathcal{E}(V) \otimes(\wedge \mathbb{V}) \simeq H(\mathcal{E}(V) ; \mathcal{E}(V))
$$

где $H_{\mathrm{diff}}(\mathcal{E}(V) ; \mathcal{E}(V))$ - пространство дифференииальных когомологий.

Благодарности. Работа выполнена при поддержке грантов РФФИ № 04-01-00769 и НШ-1542.2003.1

\section{Список литературы}

[1] G. Hochschild. Ann. Math. 1948. V. 46. № 1. P. 58-67.

[2] C. Chevalley, E. Eilenberg. Trans. Am. Math. Soc. 1948. V. 63. P. 85-124.

[3] M. Gerstenhaber. Ann. Math. 1964. V. 79. № 1. P. 59-103; 1966. V. 84. № 1. P. 1-19; 1968. V. 88. № 1. P. 1-34.

[4] M. Gerstenhaber. Ann. Math. 1974. V. 99. P. 257-276.

[5] F. Baen, M. Flato, C. Fronsdal, A. Lichnerowicz, D. Sternheimer. Ann. Phys. 1978. V. 111. P. $61-110 ;$ P. 111-151.

[6] G. Dito, D. Sternheimer. Deformation quantization: genesis, developments and metamorphoses. In: Deformation Quantization. IRMA Lect. in Math. and Theor. Phys. V. 1. Ed. G. Halbout. Berlin: de Gruyter, 2002. P. 9-54; math.QA/0201168.

[7] J. L. Taylor. Adv. Math. 1972. V. 9. P. 137-182.

[8] В. В. ЖКаринов. ТМФ. 2001. Т. 128. № 2. С. 147-160; 2003. Т. 136. № 2. С. 179-196.

[9] Л. Хермандер. Анализ линейных дифференциальных операторов с частными производными. Т. 1. Теория распределений и анализ Фурье. М.: Мир, 1986.

[10] J. Vey. Comment. Math. Helv. 1975. V. 50. P. 421-454; S. Gutt. Lett. Math. Phys. 1997. V. 39. P. 157-162. 